\title{
Rapid Efficacy of Ixekizumab for Pediatric Acute Generalized Pustular Psoriasis
}

\author{
Linda Serrano, MD'1, Valerie Carlberg, MD ${ }^{1,2}$, Kristen Holland, MD ${ }^{1,2}$ \\ ${ }^{1}$ Medical College of Wisconsin, Department of Dermatology, Milwaukee WI \\ ${ }^{2}$ Children's Hospital of Wisconsin, Department of Dermatology, Milwaukee WI
}

\section{ABSTRACT}

Introduction: Generalized pustular psoriasis (GPP) is a rare, severe variant of psoriasis that is uncommon in pediatric patients and can be refractory to many therapies. Ixekizumab, a monoclonal antibody that selectively inhibits interleukin 17-A, has been reported as safe and efficacious in patients with GPP, though minimal data exists on its use in the pediatric population.

Case presentation: A 17-year-old female with a history of alopecia areata and pustular psoriasis, on ustekinumab, was admitted for a severe pustular psoriasis flare with systemic symptoms including fever and tachycardia which progressed to erythroderma and required vasopressor support. After minimal improvement with 24 hours on broad-spectrum antibiotics, she received cyclosporine $5 \mathrm{mg} / \mathrm{kg} / \mathrm{day}$. On day three of admission, and after two days of cyclosporine with minimal improvement, she received a $160 \mathrm{mg}$ loading dose of ixekizumab. She defervesced and transferred out of the ICU within 24 hours. She was successfully weaned off cyclosporine after her second dose of ixekizumab. She experienced no adverse reactions.

Discussion: Generalized pustular psoriasis is less common and often more severe than plaque psoriasis, associated with increased morbidity and mortality in both pediatric and adult patients.

Clinical trials and case series have reported rapid and sustained improvement in patients with pustular psoriasis refractory to other therapies, though there is little data on the pediatric population. This case demonstrates the rapid efficacy of ixekizumab for severe erythrodermic pustular psoriasis in a pediatric patient, highlighting its use not only for refractory disease, but also as a rescue therapy in an emergent setting.

\section{INTRODUCTION}

Generalized pustular psoriasis (GPP) is an uncommon life-threatening subtype of psoriasis which is characterized by widespread sterile pustules overlying erythematous plaques. 1,2 GPP has a female predominance with a median age of onset during the fifth decade of life, though earlier onset is noted in patients with a family history of psoriasis or homozygous mutation of Interleukin (IL) 36RN. ${ }^{3}$ IL36RN and CARD14 are genes which encode proteins secreted by keratinocytes and are thought to contribute to the susceptibility of GPP. 1,4 GPP accounts for $0.6 \%$ to $7 \%$ pediatric psoriasis cases, with a male predominance and onset between 3 and 16 years of age. ${ }^{3}$ GPP may be precipitated by infection, pregnancy, hypocalcemia associated with hypothyroidism, and drugs. 1 Patients often present with features of sepsis and a coexisting infection which can lead to both diagnostic and therapeutic challenges. ${ }^{5}$ Systemic manifestations of GPP include neutrophilic cholangitis, cholestasis, epigastric pain, otitis media, arthritis, interstitial pneumonitis, and renal failure. ${ }^{3}$ 
Retinoids, cyclosporine, and methotrexate, have shown efficacy in GPP, however, the use of these drugs presents a risk of toxicity. ${ }^{2}$ We herein report a case of refractory GPP treated with ixekizumab, a high affinity monoclonal antibody that selectively inhibits IL-17A, 6 with rapid improvement in a pediatric patient.

\section{CASE REPORT}

A 17-year-old female with history of alopecia areata and pustular psoriasis, on ustekinumab, an IL12 and 23 inhibitor, was admitted for a severe pustular psoriasis flare in the setting of dysuria and general malaise. She has a history of multiple pustular psoriasis flares in the past with known triggers including surgery and infections. She has been recalcitrant to many systemic therapies including methotrexate, cyclosporine, prednisone, infliximab, etanercept, adalimumab, and now ustekinumab. She had just returned from Mexico during the peak of the COVID-19 pandemic and her father had a current gastrointestinal illness. Additionally, she had shaved her scalp 2 days prior to presentation, in anticipation of a hair prosthesis. Examination was notable for innumerable pinpoint pustules on a background of erythema with overlying yellow crust on the scalp, trunk, and extremities including the palms and soles, with a notable predominance in the flexural surfaces (Figure 1). She had patches of alopecia with superimposed pinpoint pustules (Figure 2). There were no mucosal lesions and Nikolsky sign was negative. She had fevers, tachycardia, abdominal pain, vomiting, diarrhea, dysuria, and diffuse adenopathy.

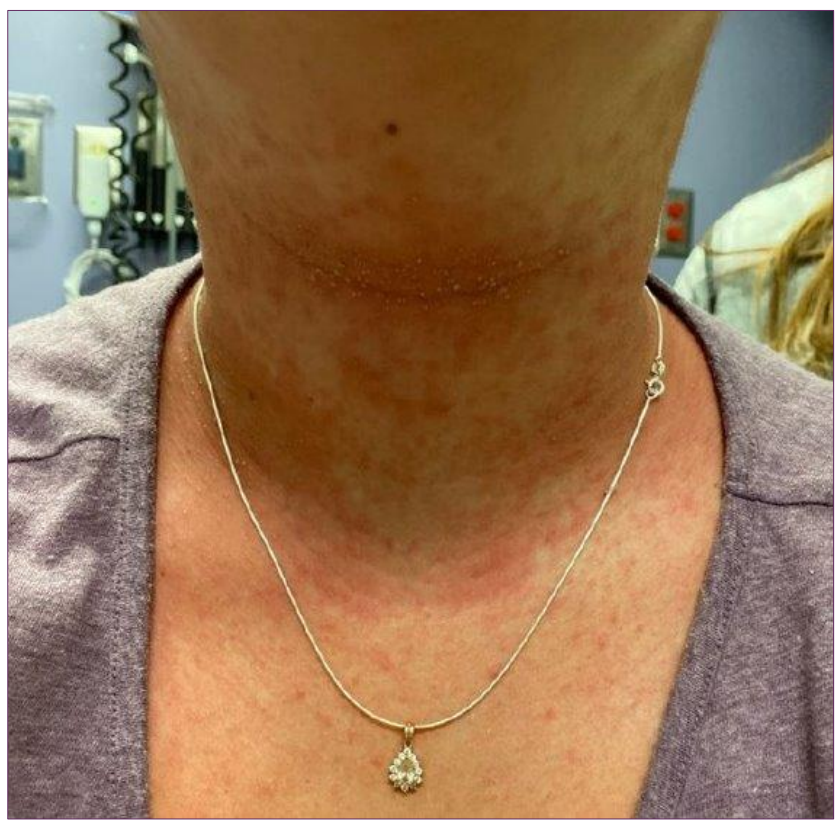

Figure 1. Initial presentation of pustular psoriasis flare with pinpoint pustules on a background of erythema located on anterior neck and chest.

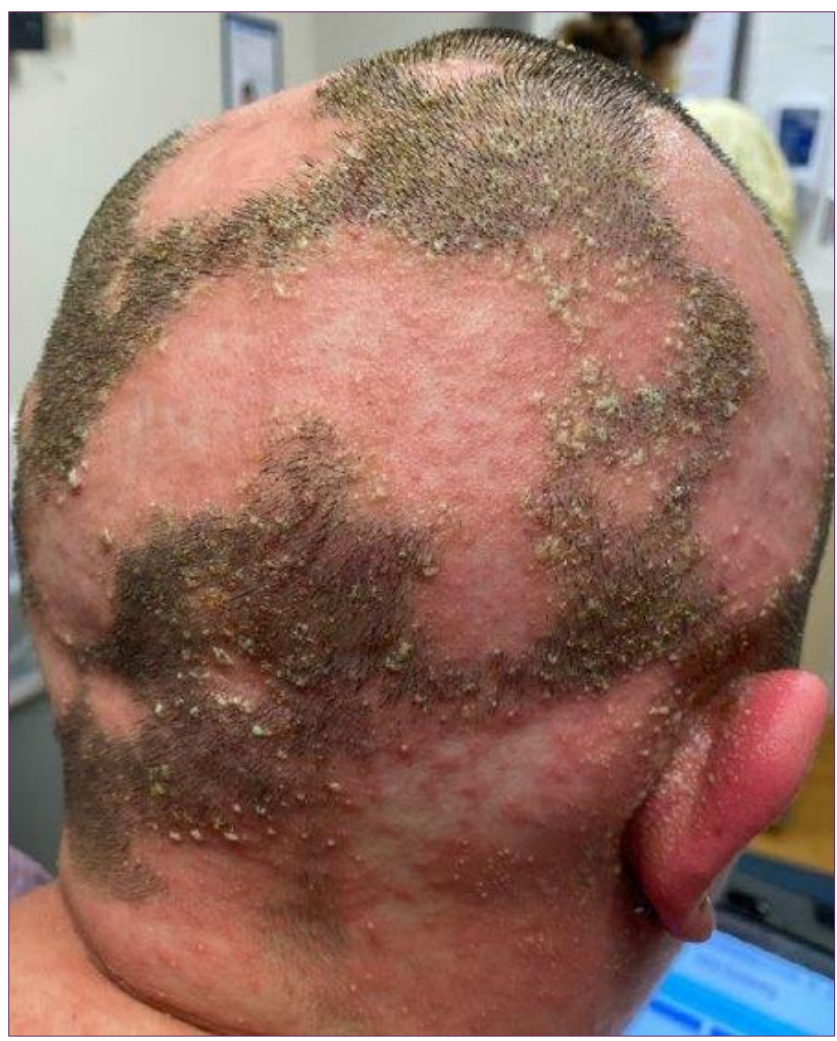

Figure 2. Initial presentation of pustular psoriasis flare with pinpoint pustules on the scalp along with patches of alopecia areata. 
Laboratory studies were notable for leukocytosis with neutrophilic predominance, elevated sedimentation rate, C-reactive protein (CRP), elevated procalcitonin (PCT), elevated transaminases, and pyuria with trace bacteria on urinalysis. These findings were concerning for both a robust inflammatory response as well as an infectious trigger. She was admitted to the general medical floor with a plan for intravenous hydration, antibiotics, and immunosuppression; however, she decompensated in a matter of hours requiring transfer to the intensive care unit (ICU) for vasopressor support. A skin biopsy was notable for a spongiotic dermatitis with a subcorneal pustule with a superficial and deep infiltrate including neutrophils and eosinophils consistent with pustular psoriasis. Her skin examination progressed to erythroderma with generalized edema and lakes of pus most concentrated on the scalp though also present diffusely (Figure 3 ).

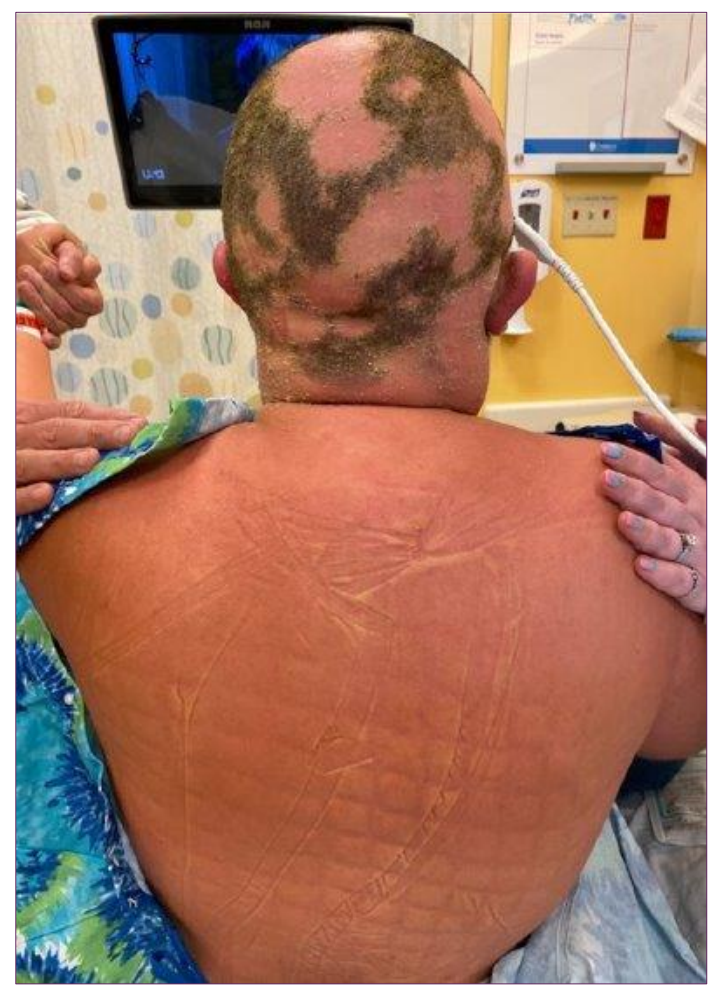

Figure 3. Progression to erythroderma day two of hospitalization.
Immunosuppression was held while infectious etiologies could be further assessed and while urgent approval of ixekizumab was sought. After minimal improvement with 24 hours of broadspectrum antibiotics, insurance barriers to inpatient biologic therapy, and negative infectious studies, she received cyclosporine $5 \mathrm{mg} / \mathrm{kg} /$ day. On day three of admission, and after two days of cyclosporine with minimal improvement, she received a $160 \mathrm{mg}$ loading dose of ixekizumab. She defervesced and transferred out of the ICU within 24 hours. Pustules desquamated, her erythema and edema decreased, and she was weaned off pain medication. She was discharged three days after starting ixekizumab and she was nearly clear at her one-week outpatient follow up (Figure 4). She was successfully weaned off cyclosporine after her second dose of ixekizumab, four weeks later, and has remained clear.

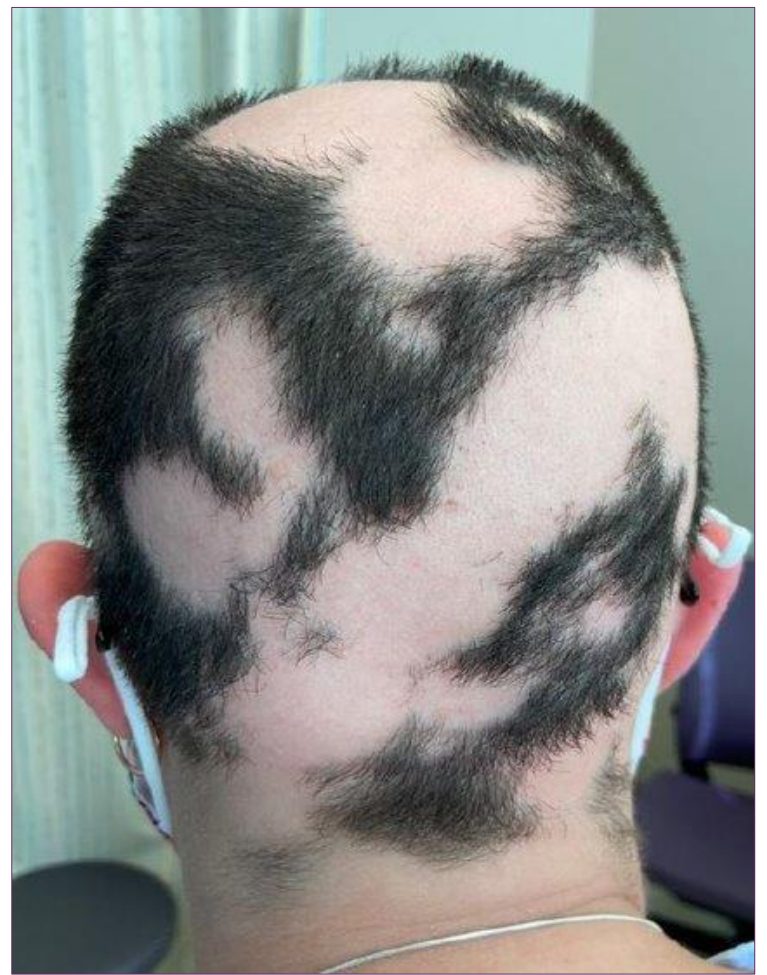

Figure 4. Near complete clearance of the scalp at one-week outpatient follow up. 


\section{DISCUSSION}

Generalized pustular psoriasis is less common and more severe than chronic plaque psoriasis and is associated with increased morbidity and mortality in pediatric and adult patients. Our patient's case was particularly challenging given her acute presentation which overlapped with septic shock. PCT when used in combination with CRP can be useful in differentiating bacterial infection from a GPP flare. A PCT of less than 1.50 along and/or a CRP of less than 46.75 can likely exclude a bacterial infection as the cause. ${ }^{5}$ Our patient had a PCT of 7.35 and a CRP of 19.9. Though the PCT was suggestive of infection, we were comfortable with higher immunosuppression based on the CRP being well below the reported cut off value for infection and given all her infectious studies came back negative. ${ }^{5}$ We sought a rapid acting therapeutic with less immunosuppressive potential than traditional therapies, of which she had failed many in the past. Ixekizumab is a high affinity monoclonal antibody that selectively inhibits interleukin 17-A. ${ }^{6}$ Clinical trials and case series have reported rapid and sustained improvement in pustular psoriasis in patients refractory to other therapies, though there is little data on the pediatric population. 6-8 Multiple cases of adolescent males with known deficiency of the interleukin IL-36 receptor antagonist (DITRA), characterized by fevers and pustular psoriasis flares, who were refractory to a variety of treatments such as acitretin, infliximab, cyclosporine, phototherapy, adalimumab, prednisone, ustekinumab, methotrexate, apremilast, and anikinara had a notable response to secukinumab, another anti- IL-17 monoclonal antibody. ${ }^{9}, 10 \mathrm{IL}-17 \mathrm{~A}$ is highly expressed in skin of patients with GPP and reportedly plays a role in the activation of neutrophils ${ }^{10}$, 11. IL-36 cytokines regulate Th17 cytokines and correlate with increased levels of IL-17. A pathomechanistic link between defective IL36RN and Th17 differentiation in DITRA has been proposed though not yet completely understood..$^{9,10,12}$

\section{CONCLUSION}

We present a 17-year old female with a severe GPP flare that rapidly improved with ixekizumab. Ixekizumab along with other IL17 inhibitors have been shown to be safe and effective in GPP and should be considered for treatment in refractory pediatric cases of pustular psoriasis such as this. Further investigation is warranted in the use of IL-17 inhibitors in GPP, especially in the pediatric population.

\section{Conflict of Interest Disclosures: None}

Funding: None

Corresponding Author:

Linda Serrano MD

Medical College of Wisconsin

8701 Watertown Plank Rd

Milwaukee, WI 53226

Phone: 414-955-3122

Email: Iserrano@mcw.edu

\section{References:}

1. Sugiura K. The genetic background of generalized pustular psoriasis: IL36RN mutations and CARD14 gain-of-function variants. J Dermatol Sci 2014;74:187-92.

2. Wang WM, Jin HZ. Biologics in the treatment of pustular psoriasis. Expert Opin Drug Saf 2020;19:969-80.

3. Hoegler KM, John AM, Handler MZ, Schwartz RA. Generalized pustular psoriasis: a review and update on treatment. J Eur Acad Dermatol Venereol 2018;32:1645-51.

4. Marrakchi S, Guigue P, Renshaw BR, Puel A, Pei $X Y$, Fraitag $S$ et al. Interleukin-36-receptor antagonist deficiency and generalized pustular psoriasis. N Engl J Med 2011;365:620-8.

5. Wang S, Xie Z, Shen Z. Serum procalcitonin and C-reactive protein in the evaluation of bacterial 
infection in generalized pustular psoriasis. An

Bras Dermatol 2019;94:542-8.

6. Okubo Y, Mabuchi T, Iwatsuki K, Elmaraghy H, Torisu-Itakura $\mathrm{H}$, Morisaki $\mathrm{Y}$ et al. Long-term efficacy and safety of ixekizumab in Japanese patients with erythrodermic or generalized pustular psoriasis: subgroup analyses of an open-label, phase 3 study (UNCOVER-J). J Eur Acad Dermatol Venereol 2019;33:325-32.

7. Nagata M, Kamata M, Fukaya S, Hayashi K, Fukuyasu A, Tanaka T et al. Real-world singlecenter experience with 10 cases of generalized pustular psoriasis successfully treated with ixekizumab. J Am Acad Dermatol 2020;82:75861.

8. Saeki H, Nakagawa H, Nakajo K, Ishii T, Morisaki $\mathrm{Y}$, Aoki T et al. Efficacy and safety of ixekizumab treatment for Japanese patients with moderate to severe plaque psoriasis, erythrodermic psoriasis and generalized pustular psoriasis: Results from a 52-week, open-label, phase 3 study (UNCOVER-J). J Dermatol 2017;44:355-62.

9. Cordoro KM, Ucmak D, Hitraya-Low M, Rosenblum MD , Liao W. Response to Interleukin (IL)-17 Inhibition in an Adolescent With Severe Manifestations of IL-36 Receptor Antagonist Deficiency (DITRA). JAMA Dermatology 2017;153:106-8.

10. Molho-Pessach V, Alyan R, Gordon D, Jaradat H , Zlotogorski A. Secukinumab for the Treatment of Deficiency of Interleukin 36 Receptor Antagonist in an Adolescent. JAMA Dermatol 2017;153:473-5.

11. Yilmaz SB, Cicek N, Coskun M, Yegin O, Alpsoy E. Serum and tissue levels of IL-17 in different clinical subtypes of psoriasis. Arch Dermatol Res 2012;304:465-9.

12. Carrier $\mathrm{Y}, \mathrm{Ma} \mathrm{HL}$, Ramon HE, Napierata $\mathrm{L}$, Small $\mathrm{C}$, O'Toole $\mathrm{M}$ et al. Inter-regulation of Th17 cytokines and the IL-36 cytokines in vitro and in vivo: implications in psoriasis pathogenesis. $\mathrm{J}$ Invest Dermatol 2011;131:2428-37. 